\title{
Enterocele tardia pós histerectomia: relato de caso
}

\author{
Late enterocele after hysterectomy: case report
}

Enterocele tardío después de la histerectomia: reporte de un caso

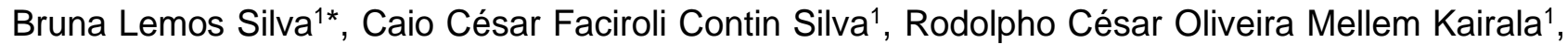
Eduardo Reinaldo Silva², Lucas Costa Corgozinho², Maurício Adriano Trentini Bertolin², Patrick Rogério Carvalhaes Santos ${ }^{2}$, Vinícius de Oliveira Silva².

\section{RESUMO}

Objetivo: Apresentação de quadro clínico de uma paciente que apresentou prolapso de órgão pélvico, no caso enterocele. Detalhamento do caso: Trata-se de um relato de caso a partir de análise de prontuário de uma paciente de 85 anos, submetida a cirurgia de correção de enterocele. O procedimento ocorreu no ano de 2019, em um hospital público localizado em uma cidade do interior do estado de São Paulo. Levando-se em consideração a idade avançada, a sintomatologia apresentada e o grau de deiscência do órgão foi sugerido a paciente as classes terapêuticas ideais para tratamento. Logo, foi acordado com ela e com a equipe de saúde que seria cirúrgico. Considerações finais: Por conseguinte, conclui-se que a enterocele é um tipo de prolapso de órgão pélvico. Outrossim, possui alta taxa de prevalência a qual aumenta de acordo com a idade da mulher. Contudo, o tratamento deve ser analisado junto a paciente, respeitando seus anseios, comorbidades e necessidades. A fim de, optar pela modalidade cirúrgica ou conservadora.

Palavras-chave: Prolapso de órgão pélvico, Enterocele, Hérnia, Prolapso.

\begin{abstract}
Objective: Presentation of a clinical picture of a patient who had pelvic organ prolapse, in the case of enterocele. Case Details: This is a case report from the analysis of the medical records of an 84-year-old patient who underwent surgery to correct the enterocele. The procedure took place in 2019, in a public hospital located in a city in the interior of the state of São Paulo. Taking into account the advanced age, the presented symptoms and the degree of dehiscence of the organ, the therapeutic classes ideal for treatment were suggested to the patient. So, it was agreed with her and the health team that it would be surgical. Final considerations: Therefore, it is concluded that the enterocele is a type of pelvic organ prolapse. Furthermore, it has a high prevalence rate which increases according to the woman's age. However, the treatment must be analyzed with the patient, respecting their wishes, comorbidities and needs. In order to, choose the surgical or conservative modality.
\end{abstract}

Key words: Pelvic organ prolapse, Enterocele, Hernia, Prolapse.

\section{RESUMEN}

Objetivo: Presentación de un cuadro clínico de una paciente con prolapso de órganos pélvicos, en el caso del enterocele. Detalles del caso: Este es un informe de caso basado en el análisis de los registros médicos de un paciente de 84 años que se sometió a cirugía para corregir el enterocele. El procedimiento tuvo lugar en 2019, en un hospital público ubicado en una ciudad del interior del estado de São Paulo. Teniendo en cuenta la edad avanzada, los síntomas presentados y el grado de dehiscencia de los órganos, se sugirieron

${ }^{1}$ Santa Casa de Misericórdia de Franca, Franca - SP. *E-mail: blemosilva@gmail.com

2 Universidade de Franca (UNIFRAN), Franca - SP. 
al paciente las clases terapéuticas ideales para el tratamiento. Entonces, se acordó con ella y el equipo de salud que sería quirúrgico. Consideraciones finales: Por lo tanto, se concluye que el enterocele es un tipo de prolapso de órganos pélvicos. Además, tiene una alta tasa de prevalencia que aumenta según la edad de la mujer. Sin embargo, el tratamiento debe analizarse con el paciente, respetando sus deseos, comorbilidades y necesidades. A fin de, elegir la modalidad quirúrgica o conservadora.

Palabras clave: Prolapso de órganos pélvicos, Enterocele, Hernia, Prolapso.

\section{INTRODUÇÃO}

A enterocele pode ser definida como o prolapso de órgãos intraperitoneais por meio da vagina. Tal acontecimento é comum. Ademais, apesar de possuir baixo grau de morbimortalidade, pode produzir intenso desconforto a mulher. Logo que, as manifestações de tal patologia influem em seu cotidiano, em sua sexualidade e em sua prática de atividade física. Assim, tal quadro merece importante relevância dentro da ciência médica (HORST W e SILVA J, 2016).

O prolapso de órgãos pélvicos (POP) entidade na qual está inserida a enterocele apresenta pequena taxa de complicações. Porém, alta incidência na população geral feminina, o que aumenta o número de procedimentos cirúrgicos para correção, consequentemente onera os sistemas de saúde (BARBER MD e MAHER C, 2013).

O POP é uma patologia frequente nos indivíduos do sexo feminino de uma maneira geral. Porquanto, estima-se que cerca de $40 \%$ de todas as mulheres serão acometidas por tal alteração anatômica. Ademais, a incidência é diretamente proporcional ao envelhecimento populacional, logo que tem sido verificado que com o aumento da expectativa de vida, os casos de POP também têm aumentado. Em vista disso, $30 \%$ das mulheres com idades entre 50 e 89 anos procuram atendimento médico anualmente, graças a essa comorbidade (GIANNINI A, et al., 2019).

Essa alteração anatômica pode proporcionar alguns sinais e sintomas desconfortáveis os quais levam a diminuição da qualidade de vida, assim como intensificam suas comorbidades. Dentre tais manifestações, pode-se citar o abaulamento físico o qual inclusive pode estar presente além do introito vaginal. Outrossim, disfunção defecatória, dor e eventual sangramento (TOWERS G, et al., 2020).

Em vista do aumento na prevalência dos POPs com o envelhecer da população, associado a intensa interferência na qualidade de vida das mulheres acometidas. Bem como, as taxas não muito relevantes de correção desta alteração com as terapias disponíveis atualmente, esse assunto tem sido bastante discutido por profissionais e pesquisadores da área (DELANCEY JOL, 2005).

Apesar de não existir uma terapêutica ideal para correção dos POPs, o objetivo principal das existentes é a atenuação da sintomatologia. Em cada caso, deve-se levar em consideração a singularidade do paciente, quais são suas ambições em relação a conclusão da intervenção médica e a influência na qualidade de vida. Assim, pode-se oferecer a todas as mulheres sintomáticas possibilidades de tratamentos conservadores ou cirúrgicos (JELOVSEK JE, et al., 2007).

Nessa conformidade, a escolha do tipo de tratamento se baseará nas demais comorbidades apresentadas pelas pacientes, na idade, na sintomatologia, no grau de deiscência dos órgãos pélvicos. Ademais, ao risco cardiovascular do indivíduo em questão, afinal pacientes com alto risco têm mais probabilidade de complicações cirúrgicas. Por fim, a opinião e anseios da paciente (LUBER KM, et al., 2001).

Diante tais dados, o objetivo deste estudo é evidenciar o quadro clínico de uma paciente a qual apresentava POP, definida como enterocele. Da mesma maneira que, expor as informações mais atuais e confiáveis sobre tal comorbidade. Assim, contribuindo com novos trabalhos e com o meio acadêmico. Logo que, tal patologia tem alta frequência, portanto quanto mais descrições de pacientes e do tratamento efetuado, existe a possibilidade de aprimoramento do cuidado. 


\section{DETALHAMENTO DO CASO}

Trata-se de um relato de caso, construído a partir de análise de prontuário eletrônico de uma paciente submetida à tratamento cirúrgico para a correção de enterocele. Tal procedimento ocorreu no ano de 2019, em um hospital público localizado em uma cidade do interior do estado de São Paulo.

Os dados relatados aqui foram colhidos de prontuário do hospital mencionado por residentes da especialidade cirurgia geral e internos do curso de medicina, de acordo com a disponibilidade de horário de cada um, sob a autorização da direção clínica do hospital.

Foi realizado contato entre os pesquisadores e a paciente cujo caso é aqui redigido, a partir do endereço que consta no prontuário. Assim, foi permito por ela a criação e publicação deste artigo. Também, este trabalho foi aprovado pelo Comitê de Ética em Pesquisa, protocolo CAAE N 26910719.0.0000.5438 e obedeceu aos princípios éticos e legais da resolução no 466/12.

A participante assinou de maneira voluntária o Termo de Consentimento Livre e Esclarecido, após a leitura deste documento por ela. Ademais, ainda antes de assinar, os pesquisadores também explicaram a ela sobre o que tratava tal estudo, assim como os seus potenciais riscos.

Os dados desta pesquisa foram colhidos de paciente feminina com 85 anos, viúva, cor parda e brasileira. Há 5 anos antes do surgimento da enterocele, ela apresentava queixa de sangramento uterino anormal e anemia sem melhora com tratamento hormonal e suplementação de ferro. Por isso, foi realizada histerectomia via vaginal, em virtude do diagnóstico de miomatose uteriana com repercussão sistêmica. A cirurgia transcorreu sem intercorrências. Ademais, a paciente não apresentou complicações no pós-operatório (PO) imediato e recebeu alta hospitalar após 48 horas de internação.

Todavia, após 5 anos do procedimento cirúrgico, procurou atendimento médico devido à grande abaulamento o qual se apresentava exteriorizado a abertura vaginal, associado a alterações de função intestinal e algia de caráter constante de localização imprecisa, mas em hipogástrio, de surgimento em poucos minutos e moderada intensidade. Consequentemente, foi diagnosticada com enterocele pelo canal vaginal, na qual estavam presentes alças de delgado as quais estavam viáveis, levando-se em consideração a perfusão tecidual. Análise a qual foi confirmada por meio de laparotomia exploradora. Após isso, foi realizado então cirurgia para correção. Para o reparo de cúpula vaginal foi utilizando Vicryl 1-0, sutura com peritônio em uma segunda linha e uma sutura complementar via vaginal. A cirurgia transcorreu sem intercorrências. A paciente não apresentou complicações no PO imediato.

No PO foi prescrito ceftriaxona e metronidazol por 4 dias, mantendo paciente em jejum e sonda vesical de demora (SVD) por 24hs. No segundo dia de PO, retirou-se a SVD, prescrita dieta líquida restrita permanecendo internada por mais um dia, com boa evolução no dia posterior sendo liberada dieta líquida completa, sem complicações. Sendo que, no dia seguinte a paciente recebeu alta hospitalar após 72 horas de internação, com boa aceitação da dieta e melhora dos sintomas, ademais foi prescrito ciprofloxacino e metronidazol por mais 3 dias.

Deste modo, ao considerar as etapas do procedimento descrito, assim como os dados técnicos presentes em literatura médica atual, pode-se dizer que tal ato cirúrgico foi realizado de maneira apropriada para a situação vigente. Assim como, pode-se inferir que é uma situação frequente e que na maioria dos casos exige correção cirúrgica, para sua completa resolução.

\section{DISCUSSÃO}

O prolapso genital é a ptose de uma região da parede vaginal que pode ser a anterior, posterior ou o ápice, está última sendo relacionada a descida uterina, ou da cúpula vaginal após histerectomia. Outrossim, a enterocele é comumente associada a este tipo de procedimento cirúrgico (HORST W e SILVA J, 2016; LIMA MIM, et al., 2012).

A saúde é uma circunstância na qual o indivíduo vivencia completo bem-estar físico, mental e social. Portanto, uma paciente a qual apresente enterocele pode ser considerada uma pessoa a qual não é 
privilegiada por saúde. Uma vez que, apresenta sinais e sintomas os quais influem em sua qualidade de vida. Outrossim, aquelas que ainda não apresentam sintomatologia, possivelmente passarão a apresentar com a evolução da alteração anatômica (ORGANIZAÇÃO PAN-AMERICANA DA SAÚDE, 2020; BARBER MD e MAHER C, 2013).

O POP quando pesquisado apenas pela sintomatologia, apresenta taxa de prevalência que varia entre 3 e $6 \%$. Entretanto, quando investigado pelo exame ginecológico, pode-se obter taxa de prevalência de até $50 \%$. Em virtude de tal valor elevado, o número de procedimentos realizados para sua correção também é alto. São realizadas duas vezes mais cirurgias para correção de POP do que para reparo de incontinência urinária. Em resumo, os procedimentos realizados por ano para retificação da POP variam entre 1,5 a 1,8 por 1.000 mulheres, sendo que a faixa etária mais prevalente submetida é entre 60 e 69 anos (BARBER MD e MAHER C, 2013).

O POP quando sintomático pode ser referido pelas mulheres acometidas como sensação de pressão pélvica, abaulamento vaginal, ou sintomas sexuais, intestinais ou urinários. Quanto a alteração urinária, relatam incontinência urinária de esforço, ou até mesmo sintomas urinários obstrutivos, caracterizados por sensação de esvaziamento vesical incompleto, intermitência, hesitação e retenção urinária (FEBRASGO, 2015).

Já em relação aos sinais e sintomas intestinais, elas podem afirmar apresentar obstipação intestinal, urgência, sensação de esvaziamento incompleto e necessidade de digitopressão para auxiliar no ato defecatório. Portanto, o quadro clínico pode ser bastante desconfortável (FEBRASGO, 2015).

O POP é uma situação desencadeada por inúmeros fatores. Dentre eles, pode ser citado o parto vaginal, este sendo o principal determinante. Porém, também estão associados: hipoestrogenismo, macrossomia fetal, uso de fórcipe e vácuo-extrator, episiotomia, cirurgias prévias para correção de distopia genital e histerectomia. Sendo esta, provavelmente, o principal fator desencadeante da enterocele apresentada pela paciente deste estudo (RODRIGUES AM, et al., 2009; MANT J, et al., 1997; DELANCEY JOL, 2005).

Ainda, existem trabalhos os quais relatam outros fatores, como o avançar da idade, a obesidade e fatores étnicos, pois mulheres asiáticas e caucasianas têm menores chances, quando comparadas com hispânicas. Também, podem ser considerados as doenças do colágeno e até mesmo fatores relacionados com esforço crônico, como a constipação crônica, levantamento de peso e tosse crônica (HORST W e SILVA J, 2016).

O assoalho pélvico da mulher é uma estrutura complexa a qual é composta por músculos os quais funcionam como uma única unidade. Consequentemente, é possível desempenhar a função de suporte aos órgãos presentes no espaço pélvico e também fechar a abertura pélvica durante uma contração ou aumento de pressão na região. Por consequência, evita a perda involuntária de conteúdo fecal e de urina. Dessarte, procedimentos cirúrgicos neste local podem levar a alteração anatômica, pois invariavelmente danos a vascularização de tais músculos é produzida, levando a alterações isquêmicas, mesmo que mínimas. Assim, repercutindo na funcionalidade desta estrutura (PIVETTA HMF, et al., 2014).

Alguns autores associam a realização de histerectomia com o surgimento de disfunções do aparelho urinário, genital e anorretal. Sendo que, as alterações mais frequentes associadas são prolapso genital, dano a inervação das vísceras pélvicas e modificação da distribuição dos vasos sanguíneos da região. Assim, favorecendo ao surgimento de POP a qual poderia ser do tipo enterocele (PIVETTA HMF, et al., 2014).

A histerectomia é uma cirurgia na qual consiste na exérese uterina. Tal ato está entre os procedimentos cirúrgicos mais realizados em indivíduos femininos que se encontram em idade reprodutiva. Visto que, cerca de $30 \%$ delas passaram por tal procedimento, além disso são realizadas cerca de 300 mil indicações de histerectomia por ano, no Brasil (PIVETTA HMF, et al., 2014).

Quanto ao tratamento do POP, existem métodos cirúrgicos e conservadores. Em relação ao procedimento cirúrgico curativo da POP, objetiva-se a recuperação da anatomia da parede vaginal. Dessa forma, podendo restaurar a funcionalidade dos órgãos abdominais que estejam dentro da enterocele. Assim como, diminuição da sintomatologia apresentada pela paciente (SIDDIQUI NY e EDENFIELD AL, 2014). 
A escolha da técnica depende do melhor acesso para a correção, instrumentos disponíveis e da experiência do cirurgião. Pode ser prudente a combinação de acessos, para garantir a reconstrução das áreas afetadas. Ademais, se associado ao prolapso coexistir incontinência urinária ou fecal, é mandatório realizar tais correções no mesmo ato cirúrgico, para garantir melhores resultados (BARBER MD, et al., 2009; SIDDIQUI NY e EDENFIELD AL, 2014).

Também, há algumas opções para pacientes que apresentam contraindicações aos atos cirúrgicos, ou caso não desejam passar por procedimento invasivo. Um desses métodos são os pessários, que podem ser constituídos por próteses de látex ou silicone, possuem diversas formas e têm missão de ocupar o canal vaginal e de sustentar as estruturas pélvicas. Essa terapêutica apresenta baixo custo e raramente apresenta complicações. Entretanto, possui uma inconveniência ao uso, a retirada diária para limpeza, a fim de evitar processos infecciosos (JELOVSEK JE, et al., 2007; WEBER AM e RICHTER HE, 2005).

Diante o exposto, pode-se afirmar que a paciente em questão apresentava como fatores de risco para o surgimento de prolapso de órgão pélvico sua idade de 89 anos, hipoestrogenismo e histerectomia prévia. Por conseguinte, uma associação de condições as quais provavelmente agiram de maneira sinérgica para 0 acometimento por enterocele. Ademais, como queixado por essa paciente e evidenciado em literatura tal patologia frequentemente é relacionada a alteração de trânsito intestinal.

Destarte, em virtude da sintomatologia e do intenso abaulamento apresentado pela paciente, foi definido que o melhor tratamento para ela seria por meio de ato cirúrgico, já que seria necessária a correção anatômica da região, assim diminuindo a deiscência e sua sintomatologia. Além do que, devido a quantidade de alças intestinais presentes na enterocele e ao quadro de dor, seria necessária a análise da viabilidade do intestino durante o procedimento, em razão da possibilidade de sofrimento isquêmico de alça intestinal.

Todavia, como exposto, cada terapêutica deve ser individualizada, a fim de proporcionar a paciente a melhor opção de tratamento às características de sua patologia, assim como para atender aos seus anseios individuais. Logo, desempenhando um ato médico que vise o principal objetivo do tratamento, diminuição de sintomatologia. Também, visando a prevenção de iatrogenias.

Em vista a todas as informações apresentadas, conclui-se que a enterocele é um tipo de prolapso de órgão pélvico. Além de que, possui alta taxa de prevalência a qual aumenta de acordo com a idade da mulher, assim como com a associação de outros fatores de risco. Outrossim, para o tratamento existem duas modalidades, o cirúrgico e o conservador, sendo que a escolha será tomada por uma decisão conjunta entre a paciente e o médico. Nessa conformidade, este deve elucidar as vantagens e desvantagens de cada opção.

\section{AGRADECIMENTOS}

Agradecemos a toda equipe hospitalar a qual participou do cuidado da paciente em questão, desde o recepcionista do hospital, até o médico que liberou a alta para ela. Assim como, não podemos deixar de mencionar o agradecimento ao hospital como instituição que permitiu que esse trabalho fosse elaborado. Ademais, somos gratos a todo o setor de ensino e pesquisa dessa instituição que colaborou em todas as etapas de estudo.

\section{REFERÊNCIAS}

1. BARBER MD, et al. Defining success after surgery for pelvic organ prolapse. Obstet Gynecol, 2009; 114(3): 600-609.

2. BARBER MD, MAHER C. Epidemiology and outcome assessment of pelvic organ prolapse. International Urogynecology Journal volume, 2013; 24(11): 1783-1790.

3. DELANCEY JOL. The hidden epidemic of pelvic floor dysfunction: achievable goals for improved prevention and treatment. American Journal of Obstetrics and Gynecology, 2005; 192(5): 1488-1495.

4. FEBRASGO. Manual de uroginecologia e cirurgia vaginal. São Paulo: 2015; 8-19.

5. GIANNINI A, et al. Advances in surgical strategies for prolapse.Climacteric. Climacteric, 2019; 22(1): 60-64.

6. HORST W, SILVA J. Prolapsos de órgãos pélvicos: revisando a literatura. Arq Catarin Med, 2016; 45(2): $91-101$.

7. JELOVSEK JE, et al. Pelvic organ prolapse. Lancet, 2007; 369: 1027-1038. 
8. LIMA MIM, et al. Prolapso genital. FEMINA, 2012; 40(2): 69-77.

9. LUBER KM, et al. The demographics of pelvic floor disorders: current observations and future projections. Am J Obstet Gynecol, 2001; 184(7): 1496-1501.

10. MANT J, et al. Epidemiology of genital prolapse: observations from the Oxford Family Planning Association Study. Br J Obstet Gynaecol, 1997; 104(5): 579-585.

11. ORGANIZAÇÃO PAN-AMERICANA DA SAÚDE. In: indicadores de saúde: elementos conceituais e práticos (capítulo 1). Estados Unidos. Disponível em: https://www.paho.org/hq/index.php?option=com_content\&view=article\&id=14401: health-indicators-conceptual-andoperational-considerations-section-1\&ltemid=0\&limitstart=1\&lang=pt.

12. PIVETTA HMF, et al. Disfunções do assoalho pélvico em pacientes submetidas à histerectomia: um estudo de revisão. Cinergis, 2014; 15(1): 48-52.

13. RODRIGUES AM, et al. Fatores de risco para o prolapso genital em uma população brasileira. Rev Bras Ginecol Obstet, 2009; 31(1): 17-21.

14. SIDDIQUI NY, EDENFIELD AL. Clinical challenges in the management of vaginal prolapse. Int J Womens Health, $2014 ; 16(6): 83-94$.

15. SUBAK LL, et al. Cost of pelvic organ prolapse surgery in the United States. Obstetrics \& Gynecology, 2001; 98(4): 646-651.

16. TOWERS G, et al. Enterocele presenting as anterior rectal prolapse: resolution with vaginal repair. Obstetrics and Gynecology, 2020; 20: 1-4.

17. WEBER AM, RICHTER HE. Pelvic organ prolapse. Obstet Gynecol, 2005; 106(3): 615-634. 